\title{
Avaliação do grau de integração da atenção primária à saúde com atendimento especializado no modelo de unidade local de saúde experimentado em Portugal
}

\section{Evaluation of the degree of integration of primary health care with specialized care in the model of local health unit experienced in Portugal}

\author{
Alexandre Morais Nunes \\ (D) https://orcid.org/0000-0002-6808-7769 \\ E-mail: anunesळiscsp.ulisboa.pt \\ Universidade de Lisboa. Instituto Superior de Ciências Sociais \\ e Políticas. Centro de Administração e Políticas Públicas. \\ Lisboa, Portugal.
}

\section{Resumo}

A integração da atenção primária à saúde com a atenção especializada em hospitais foi um importante desafio colocado ao sistema de saúde português. Iniciado em 1999, por intermédio de uma experiência-piloto, essa integração ocorreu com a criação de Unidades Locais de Saúde. Toda a assistência (atenção primária e especializada) na mesma região passou a depender de uma só equipe de gestão, com as mesmas regras e modelos de funcionamento. Embora o modelo tenha já vários anos e atualmente existam oito Unidades Locais de Saúde, não há pesquisas que avaliem essa transformação. Diante desse objetivo, foi encontrado um artigo publicado na revista Saúde e Sociedade que avaliou o grau de integração da atenção primária à saúde de município de pequeno porte na Rede de Atenção à Saúde, cuja metodologia de avaliação se enquadra perfeitamente no propósito do presente estudo. Assim, foi adotado esse método de avaliação, por meio de uma matriz com 25 indicadores com parâmetros de avaliação pontuados em escala de zero a três. Seguidamente, com o objetivo de mensurar o grau de integração, foram aplicados questionários aos profissionais de saúde. A pontuação final (grau de integração) foi calculada pela relação entre a soma dos pontos recebidos e esperados, multiplicado por 10. Os resultados mostram um escore nacional de integração de 
7,03, o que indica a boa integração entre atenção primária e especializada.

Palavras-chave: Integração de Sistemas; Atenção Primária à Saúde; Hospitais; Continuidade da Assistência ao Paciente; Política de Saúde.

\section{Abstract}

The integration of primary health care with the specialized care provided in hospitals was an important challenge to the Portuguese health system. Initiated in 1999 via a pilot experience, this integration took place with the creation of Local Health Units. All assistance (primary and hospital care) in the same region came to depend on a single management team, with equal rules and operating models. Although the model has several years and there are currently eight Local Health Units, the truth is that this model has not been objectively evaluated. Considering this objective, a study was published in the journal Health and Society that evaluated the degree of integration of Primary Health Care in a small city in the Health Care Network, whose evaluation methodology fits perfectly in the objective of this study. Thus, this method of evaluation was adopted by means of a matrix with 25 indicators accompanied by evaluation parameters and scored from o to 3. Questionnaires were applied to health professionals of the Local Health Units in Portugal to measure the degree of integration. The final score (degree of integration) was estimated by the ratio between the sum of the points received and expected, multiplied by 10. The results show a national integration score of 7.03 , which indicates the good integration between primary and specialized care.

Keywords: Systems Integration; Primary Health Care; Hospitals; Continuity of Patient Care; Health Policy.

\section{Introdução}

Os sistemas públicos de assistência à saúde em todo o mundo, fundamentais para garantir o direito à saúde e a proteção de doenças, são influenciados por contextos humanos, ambientais, culturais e políticos. Cada vez mais, as necessidades na área da saúde são imprevisíveis, necessitando de uma resposta conjunta para garantir a continuidade e excelência dos serviços de assistência (Santana; Costa, 2008).

$\mathrm{Na}$ literatura, são mencionados dois tipos de integração em saúde: a integração horizontal e a vertical. Os processos de integração horizontal ocorrem quando a nova entidade criada é composta por várias organizações, pela fusão entre duas ou mais instituições, que realizam o mesmo nível de assistência, visando ganhos em termos de economias de escala e poder no mercado (Santana; Costa, 2008). Por outro lado, a integração vertical pressupõe a constituição de uma única entidade que fica responsável por vários níveis de assistência, composta por organizações que se complementam e não têm as mesmas soluções (Santana; Costa, 2008). Segundo a Organização Mundial da Saúde (OMS), a integração vertical completa é aquela que combina em um só lugar as ações de prevenção, promoção, diagnóstico, tratamento e reabilitação (Gröne; Garcia-Barbero, 2001).

A integração entre os vários níveis de assistência à saúde (integração vertical) nos processos de reforma apresenta vantagens que são retratadas na literatura e que despertaram o interesse de prestadores privados, investigadores e políticos (Gröne; Garcia-Barbero, 2001), a sua importância tem sido comprovada nas obras produzidas, destacam-se as dos seguintes autores:

1. Devers et al. (1994) e Sobczak (2002), que viam na integração uma vantagem competitiva essencial para promover uma boa reforma nos sistemas de saúde;

2. Brown e McCool (1986), que entendiam a integração como uma oportunidade para o controle das despesas e para evitar a duplicação de serviços;

3. Byrne e Ashton (1999), Contandriopoulos et al. (2003) e Leichsenring (2004) destacam 
a melhoria na qualidade como resultados dos ganhos em saúde por meio de um melhor acompanhamento de cada paciente;

4. Almeida et al. (2013), com base em um estudo comparativo entre Brasil e Espanha, destaca-se o caráter integrativo como fundamental para o estabelecimento de protocolos de colaboração, com destaque no papel do médico da atenção primária à saúde;

5. Tsutsui (2014), refere que a integração de cuidados no Japão contribuiu para o maior acompanhamento e vigilância dos idosos;

6. Ahgren e Axelsson (2011) concluem que o caráter agregador da integração supera a fragmentação na prestação da atenção à saúde.

Segundo a Organização Mundial da Saúde (OMS, 2008) e a Organização Pan-Americana da Saúde (Opas, 2011), a integração de cuidados é reconhecida como uma solução para superar a falta de articulação e alguma fragmentação entre os vários níveis de assistência, pois com a sua implementação será possível ter melhores resultados em saúde, prevenir complicações como agravamento de doenças crônicas e agir com maior precaução, o que em todo o caso permitirá poupar recursos (OMS, 2008; Opas, 2011). Para acompanhar esses processos, desde 2001, existe um observatório da OMS, em Barcelona, destinado à identificação e análise das várias estratégias de integração realizadas em todo o mundo (Gröne; Garcia-Barbero, 2001).

É comum a alguns autores, como Tsutsui (2014), Ahgren e Axelsson (2011) e Tang et al. (2015), referirem nas conclusões de seus estudos que a integração tem vantagens para os usuários (menos complicações, maior proximidade, menor necessidade de recorrer à atenção especializada) e para os agentes prestadores (menos custos associados à assistência). Por outro lado, segundo Hofmarcher, Oxley e Rusticelli (2007), a não integração dificulta a continuidade dos processos além da atenção primária.

No caso português, a integração da assistência foi conseguida por meio de um modelo de gestão inovador que conciliou a assistência primária à saúde com a resposta de cuidados especializados em uma mesma entidade denominada Unidade Local de Saúde (ULS). No momento da criação da primeira unidade desse tipo, o legislador pretendia com o processo de integração oferecer aos usuários de uma determinada região uma assistência global com maior qualidade e com facilidade de comunicação, encaminhamento e acesso a exames de diagnóstico (Portugal, 1999). No modelo de ULS, a equipe da administração detém as competências de gestão estratégica em ambos os níveis de assistência. Foi, portanto, um modelo de organização inovador e integrador de serviços (Simões, 2004).

A ULS implementada pela primeira vez em Portugal no ano de 1999, integrada em um conjunto de experiências-piloto, foi influenciada pela perspectiva da nova gestão pública (Nunes, 2016). Quanto à estrutura, elas dispõem de uma equipe de gestão (denominada por conselho de administração), constituída por um presidente (gestor), dois vogais executivos (um deles formado em economia ou administração e outro indicado pela prefeitura), dois diretores clínicos (médico da atenção básica e um outro da atenção especializada, ambos com reconhecido mérito de gestão) e um enfermeiro diretor (licenciado e especialista em enfermagem com formação acadêmica de gestão). Em termos de organização, encontram-se divididas em departamentos: um da atenção primária e um que integra a atenção especializada e que tem um responsável (médico) para cada área (ambulatório, cirurgia, internação e exames de diagnóstico). Assim, a dinâmica dos processos decorre entre esses agentes que coordenam, cada um, sua equipe interdisciplinar sob a supervisão do conselho de administração. Essa comunicação é feita por meio de um sistema de informação interno que coloca todos os funcionários para comunicar entre si e com o paciente, mantendo sempre um registro e um acompanhamento clínico de proximidade.

Relativamente às vantagens da integração de cuidados que gerou a criação das ULS, a percepção do único estudo existente realizado na primeira experiência revela que as lutas entre diretores 
da atenção primária e da atenção especializada terminaram com a criação de uma única equipe de gestão, representativa de ambas as realidades e da comunidade, o que permitiu a comunicação entre as equipes, a partilha de sistemas de informação, equipamentos e recursos humanos, e a continuidade da assistência. Por exemplo, sempre que é necessário reforçar médicos ou enfermeiros, um nível de assistência pode solicitar apoio ao outro (ULS Matosinhos, 200o). Assim, essa primeira experiência portuguesa foi ao encontro das vantagens referidas na literatura, como a vantagem competitiva citada por Sobczak (2002), a gestão eficiente dos serviços defendida por Brown e McCool (1986), o melhor acompanhamento dos pacientes como destacaram Leichsenring (2004) e Tsutsui (2014), também o fator agregador mencionado por Ahgren e Axelsson (2011) e a valorização do papel do médico da atenção primária com o encaminhamento direto para a atenção especializada como proferiu Almeida et al. (2013).

Com base nesses resultados, o modelo de ULS foi alargado na região Norte e em outras regiões do país:

1. 2011 - Unidade Local de Saúde do Nordeste (Região Norte);

2. 2008 - Unidade Local de Saúde do Alto Minho (Região Norte);

3. 2008 - Unidade Local de Saúde da Guarda (Região Centro);

4. 2009 - Unidade Local de Saúde de Castelo Branco (Região Centro);

5. 2008 - Unidade Local de Saúde do Baixo Alentejo (Região do Alentejo);

6. 2012 - Unidade Local de Saúde do Litoral Alentejano (Região do Alentejo);

7. 2007 - Unidade Local de Saúde do Norte Alentejano (Região do Alentejo).

Em Portugal, a entidade reguladora da saúde fez uma avaliação qualitativa da criação das ULS, mas sem mensurar de forma objetiva o grau de integração, motivo que originou a presente pesquisa, a fim de mensurar o grau de integração da atenção primária e especializada (hospitalar) em Portugal nas oito unidades.

Essa avaliação contribuirá para o conhecimento global sobre o tema, permitirá partilhar a experiência portuguesa e colaborará para a melhoria do atual modelo de forma a atingir uma integração completa e efetiva.

\section{Método}

Para mensurar o grau de integração, este estudo adotou o modelo criado por Wilson Vieira e Andréa Gazzinelli (2017) usado na avaliação do grau de integração da atenção primária à saúde de município de pequeno porte na Rede de Atenção à Saúde, publicado na revista Saúde e Sociedade, dado que este modelo apresenta uma matriz bem construída que permite quantificar uma matéria mais do foro qualitativo pela parametrização, que realiza pontuando intervalos percentuais dos indicadores. Contudo, foram realizadas algumas adaptações à realidade portuguesa, sendo dada ênfase à relação entre a atenção primária à saúde e a atenção especializada, de acordo com a informação disponibilizada pelas entidades estudadas.

O estudo foi desenvolvido em todas as ULS, abrangendo uma população total aproximada de 1.163.550 habitantes (INE, 2018).

Como parte do processo de adaptação da matriz, realizou-se um pré-teste (consistiu na experimentação de indicadores existentes), fundamental para realizar as adaptações necessárias e analisar o tempo necessário. Partindo da matriz de avaliação originalmente realizada por Vieira e Gazzinelli (2017), que continha 25 indicadores organizados em 12 critérios, foi construída uma nova com 18 indicadores e segundo nove critérios. Tal como na matriz original, para cada um dos indicadores foram estabelecidos parâmetros considerando uma pontuação de zero a três pontos com o objetivo de possibilitar a formulação do juízo de valor (Quadro 1). 

Quadro I - Matriz de avaliação do grau de integração da atenção primária à saúde e atenção especializada nas
Unidades Locais de Saúde em Portugal

\begin{tabular}{|c|c|c|c|}
\hline Número & Critério & Indicador & Parâmetros \\
\hline \multirow{3}{*}{ I. } & \multirow{3}{*}{$\begin{array}{l}\text { Capacidade } \\
\text { resolutiva } \\
\text { na atenção } \\
\text { primária }\end{array}$} & $\begin{array}{l}\text { Ia. Percentual de profissionais da } \\
\text { atenção primária que consideram } \\
\text { adequado o tempo de espera por } \\
\text { exames necessários }\end{array}$ & $\begin{array}{l}\text { o ponto: se } 25 \% \\
\text { I ponto: " } 25 \% 50 \% \\
2 \text { pontos: " } 50 \text { a } 75 \% \\
3 \text { pontos: } " 75 \%\end{array}$ \\
\hline & & $\begin{array}{l}\text { Ib. Catálogo de exames de } \\
\text { diagnóstico solicitados na atenção } \\
\text { primária }\end{array}$ & $\begin{array}{l}\text { o ponto: nenhum tipo de exame } \\
\text { I ponto: somente exames laboratoriais } \\
\text { básicos } \\
2 \text { pontos: exames laboratoriais básicos e de } \\
\text { média complexidade } \\
3 \text { pontos: ampliação do catálogo de } \\
\text { provas solicitadas }\end{array}$ \\
\hline & & $\begin{array}{l}\text { Ic. Coleta de amostras para exames } \\
\text { laboratoriais frequentemente } \\
\text { solicitados na atenção primária }\end{array}$ & $\begin{array}{l}\text { o ponto: não é realizada } \\
\text { I ponto: realizada um só, um dia por } \\
\text { semana } \\
2 \text { pontos: realizada na maioria dos dias } \\
\text { da semana } \\
3 \text { pontos: realizada diariamente }\end{array}$ \\
\hline 2. & $\begin{array}{l}\text { Apoio às } \\
\text { equipes de } \\
\text { atenção } \\
\text { primária }\end{array}$ & $\begin{array}{l}\text { 2a. Equipe interdisciplinar de apoio } \\
\text { na atenção primária }\end{array}$ & $\begin{array}{l}\text { o ponto: não existe } \\
\text { I ponto: existe apenas para grupos } \\
\text { específicos } \\
2 \text { pontos: existe equipe que atua como } \\
\text { referência, mas sem discussões clínicas } \\
3 \text { pontos: existe equipe que atua como } \\
\text { referência e participa em discussões clínicas }\end{array}$ \\
\hline 3. & $\begin{array}{l}\text { Atendimento } \\
\text { à demanda } \\
\text { espontânea } \\
\text { na atenção } \\
\text { primária }\end{array}$ & $\begin{array}{l}\text { 3a. Funcionamento no atendimento } \\
\text { à demanda espontânea }\end{array}$ & $\begin{array}{l}\text { o ponto: não há tolerância para consultas } \\
\text { não agendadas } \\
\text { I ponto: há tolerância para consultas não } \\
\text { agendadas apenas um dia por semana } \\
2 \text { pontos: há tolerância para consultas não } \\
\text { agendadas na maioria dos dias da semana } \\
3 \text { pontos: há sempre tolerância para } \\
\text { consultas não agendadas }\end{array}$ \\
\hline 4. & $\begin{array}{l}\text { Organização } \\
\text { da porta de } \\
\text { entrada aos } \\
\text { serviços de } \\
\text { saúde pela } \\
\text { atenção } \\
\text { Primária }\end{array}$ & $\begin{array}{l}\text { 4a. Percentual de médicos e } \\
\text { enfermeiros que consideram que } \\
\text { na maioria das vezes os usuários } \\
\text { procuram primeiramente a } \\
\text { atenção primária para o primeiro } \\
\text { atendimento }\end{array}$ & $\begin{array}{l}\text { o ponto: se } 25 \% \\
\text { I ponto: " } 25 \% 50 \% \\
2 \text { pontos: } " 50 \text { a } 75 \% \\
3 \text { pontos: } " 75 \%\end{array}$ \\
\hline
\end{tabular}


Quadro I-Continuação

\begin{tabular}{|c|c|c|c|}
\hline Número & Critério & Indicador & Parâmetros \\
\hline \multirow{3}{*}{5.} & \multirow{3}{*}{$\begin{array}{l}\text { Controle do fluxo } \\
\text { de usuários }\end{array}$} & $\begin{array}{l}\text { 5a. Análise de encaminhamentos } \\
\text { realizados para serviços da } \\
\text { atenção especializada }\end{array}$ & $\begin{array}{l}\text { o ponto: não há } \\
\text { I ponto: há análise não sistemática de } \\
\text { alguns encaminhamentos } \\
2 \text { pontos: há análise sistemática de } \\
\text { alguns encaminhamentos } \\
3 \text { pontos: há análise sistemática de todos } \\
\text { os encaminhamentos }\end{array}$ \\
\hline & & $\begin{array}{l}\text { 5b. Monitoramento das listas de espera } \\
\text { para serviços da atenção especializada }\end{array}$ & $\begin{array}{l}\text { o ponto: não há } \\
\text { I ponto: há, mas de um modo não sistemático } \\
2 \text { pontos: há, de um modo sistemático, mas sem } \\
\text { reavaliação periódica dos casos } \\
3 \text { pontos: há, de um modo sistemático e com } \\
\text { reavaliação periódica dos casos }\end{array}$ \\
\hline & & $\begin{array}{l}\text { 5c. Monitoramento das abstenções aos } \\
\text { agendamentos para serviços da atenção } \\
\text { especializada }\end{array}$ & $\begin{array}{l}\text { o ponto: não há } \\
\text { I ponto: há, mas de um modo não sistemático } \\
2 \text { pontos: há, de um modo sistemático mas sem } \\
\text { estratégia para recuperação da vaga } \\
3 \text { pontos: há, de um modo sistemático e com } \\
\text { estratégia para recuperação da vaga }\end{array}$ \\
\hline 6. & $\begin{array}{l}\text { Articulação entre } \\
\text { os serviços da } \\
\text { atenção primária } \\
\text { e serviços } \\
\text { de urgência }\end{array}$ & $\begin{array}{l}\text { 6a. Percentual de profissionais que } \\
\text { consideram que as tentativas de } \\
\text { transferência de usuários atendidos no } \\
\text { centro de saúde para a urgência são } \\
\text { sempre exitosas }\end{array}$ & $\begin{array}{l}\text { o ponto: se } 25 \% \\
\text { I ponto: " } 25 \% 50 \% \\
2 \text { pontos: } " 50 \text { a } 75 \% \\
3 \text { pontos: } " 75 \%\end{array}$ \\
\hline 7. & $\begin{array}{l}\text { Protocolos } \\
\text { clínicos }\end{array}$ & $\begin{array}{l}\text { 7a. Existência de protocolos clínicos } \\
\text { que visam a garantir a continuidade } \\
\text { de cuidados }\end{array}$ & $\begin{array}{l}\text { o ponto: não existem } \\
\text { I ponto: existem, mas restritos à } \\
\text { atenção primária } \\
2 \text { pontos: existem, mas restritos à } \\
\text { atenção especializada } \\
3 \text { pontos: existem e são compartilhados }\end{array}$ \\
\hline \multirow{4}{*}{8.} & \multirow{4}{*}{$\begin{array}{l}\text { Transferência } \\
\text { de informação } \\
\text { sobre o usuário } \\
\text { entre os serviços } \\
\text { ou profissionais } \\
\text { de saúde }\end{array}$} & $\begin{array}{l}\text { 8a. Percentual de médicos da atenção } \\
\text { primária que sempre fornecem } \\
\text { informações escritas quando o usuário } \\
\text { é referenciado para serviços de outros } \\
\text { níveis de atenção }\end{array}$ & $\begin{array}{l}\text { o ponto: se } 25 \% \\
\text { I ponto: " } 25 \% 50 \% \\
2 \text { pontos: } " 50 \text { a } 75 \% \\
3 \text { pontos: } " 75 \%\end{array}$ \\
\hline & & $\begin{array}{l}\text { 8b. Percentual de médicos } \\
\text { especialistas que sempre enviam } \\
\text { contrarreferências após avaliarem } \\
\text { o usuário }\end{array}$ & $\begin{array}{l}\text { o ponto: se } 25 \% \\
\text { I ponto: " } 25 \% 50 \% \\
2 \text { pontos: "50 a } 75 \% \\
3 \text { pontos: } " 75 \%\end{array}$ \\
\hline & & $\begin{array}{l}\text { 8c. Percentual de encaminhamentos } \\
\text { a serviços especializados que } \\
\text { apresentam descrição detalhada do } \\
\text { motivo do encaminhamento }\end{array}$ & $\begin{array}{l}\text { o ponto: se } 25 \% \\
\text { I ponto: " } 25 \% 50 \% \\
2 \text { pontos: } " 50 \text { a } 75 \% \\
3 \text { pontos: } " 75 \%\end{array}$ \\
\hline & & $\begin{array}{l}8 \mathrm{~d} \text {. Percentual de profissionais } \\
\text { da atenção primária que recebem } \\
\text { relatório de alta hospitalar após } \\
\text { internação dos usuários }\end{array}$ & $\begin{array}{l}\text { o ponto: se } 25 \% \\
\text { I ponto: " } 25 \% 50 \% \\
2 \text { pontos: " } 50 \text { a } 75 \% \\
3 \text { pontos: } " 75 \%\end{array}$ \\
\hline
\end{tabular}


Quadro I-Continuação

\begin{tabular}{|c|c|c|c|}
\hline Número & Critério & Indicador & Parâmetros \\
\hline \multirow{3}{*}{9.} & \multirow{3}{*}{$\begin{array}{l}\text { Acesso a } \\
\text { consultas } \\
\text { médicas } \\
\text { especializadas, } \\
\text { serviço de } \\
\text { diagnóstico } \\
\text { e atenção } \\
\text { hospitalar }\end{array}$} & $\begin{array}{l}\text { 9a. Percentual de profissionais que } \\
\text { consideram adequado o tempo de } \\
\text { espera para as consultas médicas } \\
\text { especializadas necessárias na prática } \\
\text { clínica para a condução dos casos }\end{array}$ & $\begin{array}{l}\text { o ponto: se } 25 \% \\
\text { I ponto: " } 25 \% 50 \% \\
2 \text { pontos: " } 50 \text { a } 75 \% \\
3 \text { pontos: " } 75 \%\end{array}$ \\
\hline & & $\begin{array}{l}\text { 9b. Percentual de profissionais que } \\
\text { consideram adequado o tempo de } \\
\text { espera até a realização dos exames } \\
\text { especializados necessários na prática } \\
\text { clínica para a condução dos casos }\end{array}$ & $\begin{array}{l}\text { o ponto: se } 25 \% \\
\text { I ponto: " } 25 \% 50 \% \\
2 \text { pontos: " } 50 \text { a } 75 \% \\
3 \text { pontos: " } 75 \%\end{array}$ \\
\hline & & $\begin{array}{l}\text { 9c. Percentual de profissionais que } \\
\text { consideram adequado o tempo de } \\
\text { espera até a realização de cirurgias } \\
\text { eletivas necessárias na prática clínica } \\
\text { para a condução dos casos }\end{array}$ & $\begin{array}{l}\text { o ponto: se } 25 \% \\
\text { I ponto: " } 25 \% 50 \% \\
2 \text { pontos: " } 50 \text { a } 75 \% \\
3 \text { pontos: " } 75 \%\end{array}$ \\
\hline
\end{tabular}

Fonte: adaptado de Vieira e Gazzinelli (2017).

Para avaliar o grau de integração, foram aplicados questionários estruturados a profissionais de saúde (médicos e enfermeiros) que atuavam em cada uma das oito unidades locais de saúde, no período anterior e posterior à integração. Ou seja, pretendeu-se obter a opinião dos profissionais que acompanharam e viveram todo o processo. Participaram do questionário um total de 160 profissionais, sendo 92 da atenção primária (52 médicos e 40 enfermeiros) e 68 da atenção especializada hospitalar (36 médicos e 32 enfermeiros). O processo de mobilização institucional partiu da colaboração dos conselhos de administração, sendo realizado o convite aos profissionais por e-mail institucional.
Após a coleta de dados, os indicadores foram pontuados segundo os critérios definidos na matriz de pontuação e com base nela foi calculado o grau de integração da atenção primária e atenção especializada, considerando o estudo de Vieira e Gazzinelli (2017), o cálculo obtido pela razão entre o resultado observado e a soma da pontuação máxima esperada (54 pontos), multiplicado por 10.

Com base no escore obtido, a extensão da integração foi classificada com base em uma escala proposta por Vieira e Gazzinelli (2017) (Quadro 2). Relativamente aos aspectos técnicos foram respeitadas todas as normas (sigilo, confidencialidade, gravação de entrevistas e proteção de dados) existentes em Portugal.

\section{Quadro 2 - Escores e classificação para avaliar o grau de integração da atenção primária à saúde de município de pequeno porte na Rede de Atenção à Saúde}

\begin{tabular}{|l|l|}
\hline \multicolumn{1}{|l|}{ Escores } & Classificação \\
\hline $0,00-1,99$ & Muito pouca integração \\
\hline $2,00-3,99$ & Pouca integração \\
\hline $4,00-5,99$ & Moderada integração \\
\hline $6,00-7,99$ & Boa integração \\
\hline $8,00-10,00$ & Perfeita integração \\
\hline
\end{tabular}

Fonte: Adaptado de Vieira e Gazzinelli (2017) 
Os resultados para cada um dos critérios/ em todas as unidades da integração no modelo de indicadores serão apresentados em contexto global,

ULS, ou seja, considerando todas as respostas obtidas.

\section{Quadro 3 - Representação dos resultados da matriz que avaliou o grau de integração da atenção primária à saúde com a atenção especializada nas Unidades Locais de Saúde em Portugal}

\begin{tabular}{|c|c|c|}
\hline Indicador & $\begin{array}{l}\text { Pontos } \\
\text { obtidos }\end{array}$ & $\begin{array}{l}\text { Escore por } \\
\text { critério }\end{array}$ \\
\hline $\begin{array}{l}\text { Ia. Percentual de profissionais da atenção primária que consideram adequado o tempo de } \\
\text { espera por exames necessários }\end{array}$ & 2 & 6,67 \\
\hline ib. Catálogo de exames de diagnóstico solicitados na atenção primária & 2 & 6,67 \\
\hline Ic. Coleta de amostras para exames laboratoriais frequentemente solicitados na atenção primária & 0 & 0 \\
\hline 2a. Equipe interdisciplinar de apoio na atenção primária & 3 & 10 \\
\hline 3a. Funcionamento no atendimento à demanda espontânea & 2 & 6,67 \\
\hline $\begin{array}{l}\text { 4a. Percentual de médicos e enfermeiros que consideram que na maioria das vezes os usuários } \\
\text { procuram primeiramente a atenção primária para o primeiro atendimento }\end{array}$ & 0 & 0 \\
\hline 5a. Análise de encaminhamentos realizados para serviços da atenção especializada & 3 & 10 \\
\hline 5b. Monitoramento das listas de espera para serviços da atenção especializada & 3 & 10 \\
\hline 5c. Monitoramento das abstenções aos agendamentos para serviços da atenção especializada & 3 & 10 \\
\hline $\begin{array}{l}\text { 6a. Percentual de profissionais que consideram que as tentativas de transferência de usuários } \\
\text { atendidos no centro de saúde para a urgência são sempre exitosas }\end{array}$ & 3 & 10 \\
\hline 7a. Existência de protocolos clínicos que visam a garantir a continuidade de cuidados & 3 & 10 \\
\hline $\begin{array}{l}\text { 8a. Percentual de médicos da atenção primária que sempre fornecem informações escritas } \\
\text { quando o usuário é referenciado para serviços de outros níveis de atenção }\end{array}$ & 3 & 10 \\
\hline $\begin{array}{l}\text { 8b. Percentual de médicos especialistas que sempre enviam contrarreferências após avaliarem } \\
\text { o usuário }\end{array}$ & 2 & 6,67 \\
\hline $\begin{array}{l}\text { 8c. Percentual de encaminhamentos a serviços especializados que apresentam descrição } \\
\text { detalhada do motivo do encaminhamento }\end{array}$ & 2 & 6,67 \\
\hline $\begin{array}{l}\text { 8d. Percentual de profissionais da atenção primária que recebem relatório de alta hospitalar } \\
\text { após internação dos usuários }\end{array}$ & 3 & 10 \\
\hline $\begin{array}{l}\text { 9a. Percentual de profissionais que consideram adequado o tempo de espera para as consultas } \\
\text { médicas especializadas necessárias na prática clínica para a condução dos casos }\end{array}$ & 2 & 6,67 \\
\hline $\begin{array}{l}\text { 9b. Percentual de profissionais que consideram adequado o tempo de espera até a realização } \\
\text { dos exames especializados necessários na prática clínica para a condução dos casos }\end{array}$ & 2 & 6,67 \\
\hline $\begin{array}{l}\text { 9c. Percentual de profissionais que consideram adequado o tempo de espera até a realização de } \\
\text { cirurgias eletivas necessárias na prática clínica para a condução dos casos }\end{array}$ & 0 & 0 \\
\hline Total de pontos obtidos & 38 & 7,03 \\
\hline
\end{tabular}


Considerando o critério "capacidade resolutiva na atenção primária”, os dados mostram que que $66 \%$ dos profissionais consideram adequado o tempo de espera (Indicador 1a: 2 pontos) e que o catálogo de exames de diagnóstico solicitados na atenção primária existe somente para exames laboratoriais básicos e de média complexidade (Indicador 1 b: 2 pontos). Porém, todos referem que a coleta de amostras para exames laboratoriais frequentemente solicitados na atenção primária não é realizada (Indicador 1c: o pontos).

No que diz respeito à disponibilização da equipe interdisciplinar de apoio integrada na atenção primária (constituída por psicólogo, médico dentista, nutricionista, fisioterapeuta), os profissionais relatam que atuam como referência e participam em discussões clínicas e na construção de projetos terapêuticos compartilhados (Indicador 2a: 3 pontos).

Segundo os inquiridos, na atenção primária, há tolerância para consultas não agendadas de um dia por semana (dia da consulta aberta) (Indicador 3a: 2 pontos). Porém, quanto ao seu papel como porta de entrada no sistema, apenas $25 \%$ dos profissionais consideram que os usuários procuram primeiramente a atenção primária para o primeiro atendimento em vez da urgência hospitalar.

Em relação ao encaminhamento da atenção primária para os serviços da atenção especializada, todos os médicos e enfermeiros referem que no decurso do processo existe uma análise (Indicador 5a: 3 pontos), com monitoramento de modo sistemático (Indicador 5b: 3 pontos) e reavaliação periódica do índice de não comparecimento com recuperação de vaga sempre que há uma abstenção (Indicador 5c: 3 pontos).

$\mathrm{Na}$ opinião dos entrevistados, a existência de protocolos clínicos que garantam a continuidade da assistência (Indicador 7a: 3 pontos) contribuiu para o sucesso do encaminhamento. Consequentemente, $75 \%$ dos profissionais consideram que as tentativas de transferência para a urgência, quando ocorrem, são sempre exitosas (Indicador 6a: 3 pontos) e $85 \%$ dos inquiridos consideram que em qualquer encaminhamento (consulta, exame, cirurgia ou urgência) o paciente é referenciado pelo médico da atenção primária que fornece informações escritas
(Indicador 8a: 3 pontos) e recebe relatório da alta após internação (Indicador 8b: 2 pontos). Porém, o percentual de encaminhamentos da atenção especializada para a atenção primária com relatório detalhado do motivo é mencionado por $66 \%$ dos entrevistados (Indicador 8c: 2 pontos).

Em termos médios, 66\% dos entrevistados consideram adequado o tempo de espera para consultas médicas especializadas e o tempo de espera para realização de exames necessários (Indicadores 9a e 9b: 2 pontos). Porém, apenas $25 \%$ dos profissionais consideram adequado o tempo de espera por cirurgias (Indicadores 9c: 1 ponto).

O número total de pontos obtidos foi de 38 dos 54 possíveis, o que dá um escore de integração de 7,03, e representa segundo o Quadro 2 uma “Boa Integração".

\section{Discussão}

De forma global, verificou-se, com a aplicação adaptada do modelo de Vieira e Gazzinelli (2017), uma "Boa Integração" entre a atenção primária e a atenção especializada em Portugal, com destaque para um forte apoio às equipes, atendimento adequado à demanda espontânea e com um processo de encaminhamento integrado que apresenta monitoramento das listas de espera e das abstenções dos agendamentos, ressalta-se ainda a existência de protocolos clínicos que facilitam a comunicação e que permitem um encaminhamento correto com informações escritas e relatórios pormenorizados em todo o fluxo (da atenção primária para a especializada e da atenção especializada para a primária).

A melhoria do acesso foi bem-sucedida com a implementação do programa de consulta por tempo e horas, que com o recurso de uma plataforma eletrônica permite encaminhar os pedidos de consultas (Portugal, 2013), e com o desenvolvimento do Sistema Integrado de Gestão do Acesso que além de encaminhar também permite, via plataforma eletrônica, o encaminhamento e monitoramento dos pedidos de exames, consulta e cirurgias dentro da própria ULS ou em outros hospitais na proximidade (Portugal, 2017; Simões; Augusto; Fronteira, 2017).

A transferência de informação melhorou nas ULS com a implementação da interoperabilidade entre os sistemas de informação, sendo obrigatória 
a emissão de uma nota interna sempre que existe um encaminhamento ou alta da urgência, uma cirurgia ou internação. Além disso, nos casos de óbitos de pacientes, a existência de um sistema eletrônico de comunicação permite ao médico da atenção primária ter conhecimento de quando um dos pacientes da sua lista faleceu (Nunes, 2017).

Como áreas de pouca integração, os resultados do estudo revelam indicadores críticos e dificuldades relacionadas à fraca capacidade resolutiva na atenção primária (casos de baixa coleta de amostras para exames laboratoriais frequentemente solicitados na atenção primária; e fraco percentual de médicos e enfermeiros que consideram que na maioria das vezes os usuários procuram primeiramente a atenção primária para o primeiro atendimento), com a prevalência da opção dos pacientes pela atenção especializada (serviço de urgência) para o primeiro atendimento, o que vai contra as recomendações internacionais (Starfield; Shi; Macinko, 2005).

Também é enfatizado que o acesso a consultas, exames e cirurgias necessárias na prática clínica para lidar com casos em tempo hábil é uma área problemática de integração. Mas, nesse ponto, é importante sublinhar que em Portugal não é um problema específico das Unidades Locais de Saúde, mas é ligado, por um lado, à falta de médicos em determinadas regiões (urologia, ortopedia, oftalmologia, dermatologia), agravada nas regiões do interior, onde sete das oito ULS estão implantadas. Portanto, o acesso nem sempre está relacionado a questões de integração (OECD, 2018).

Como principais limitações, podem-se assinalar a existência de outros indicadores/fatores que poderão influenciar na integração; o fato, como refere Vieira e Gazzinelli(2017), certos indicadores serem concorrentes e a melhoria de uns poderem induzir os outros. Ainda, cabe destacar o fato de não se incluir a percepção dos usuários (utilizadores), o que se justificou pelo fato de se tentar analisar a integração entre os profissionais envolvidos no processo, sendo por isso questionados aspectos mais técnicos e processuais. Reconhece-se essa limitação para que em estudo futuro seja levada em conta, dado que os pacientes são os principais afetados pela fraca integração e beneficiados quando essa ocorre da melhor forma.

\section{Considerações finais}

A integração da assistência à saúde na sua dimensão vertical constitui uma resposta organizacional para os sistemas de saúde que têm todas as potencialidades amplamente reconhecidas.

A avaliação da integração de cuidados em diferentes níveis de atenção é um processo complexo, mas tida como fundamental nos trabalhos de Tsutsui (2014) e de Ahgren e Axelsson (2011). Porém, na revisão da literatura, foi achado um instrumento construído por Vieira e Gazzinelli (2017) que permitem a identificação do grau de integração e em simultâneo identificar áreas de melhoria, possibilitando a implementação de um conjunto de intervenções nesse sentido. Por todas essas vantagens, a adaptação desse modelo à realidade portuguesa proporcionou avaliar de forma quantitativa o grau de integração da experiência integradora da atenção primária com a especializada, por meio de um questionário aplicado a vários profissionais de saúde envolvidos nos processos de encaminhamento entre ambos os níveis de atenção (médicos e enfermeiros).

Mesmo com uma avaliação global positiva com escore correspondente a uma "Boa Integração", a avaliação realizada no presente estudo permitiu concluir que no processo de integração da atenção primária com atenção especializada as maiores fragilidades foram encontradas na falta de complementaridade na coleta de amostras para exames; na organização da porta de entrada dos serviços de saúde (usuários não consideram primeiro a atenção primária e dirigirem-se logo para a atenção na urgência) e no acesso com algumas limitações nos tempos de espera por consultas, exames e sobretudo cirurgias. Porém, esse último ponto não reside somente na fraca integração mas é também determinado por uma insuficiente oferta de serviços na atenção especializada.

É necessário no sistema integrado de ULS trabalhar ainda mais em equipe, reforçar, por exemplo, na atenção primária, as atividades de educação à saúde e de gestão da doença crônica, e deixar para a atenção especializada os casos mais complexos que necessitem de uma assistência mais diferenciada. Caso contrário, será gerado 
um insuficiente grau de integração que poderá levar a um vazio no modelo de Unidade Local de Saúde.

\section{Referências}

AHGREN, B.; AXELSSON, R. A decade of integration and collaboration: the development of integrated health care in Sweden 2000-2010. International Journal of Integrated Care, Utrecht, v. 11, n. 7, art. eoo7, 2011. Disponível em: <https://bit.ly/3iPPoDv>. Acesso em: 20 maio 2018.

ALMEIDA, P. et al. Estratégias de integração entre atenção primária à saúde e atenção especializada: paralelos entre Brasil e Espanha. Saúde em Debate, Rio de Janeiro, v. 37, n. 98, p. 40o-415, 2013.

BROWN, M.; MCCOOL, B. P. Vertical integration: exploration of a popular strategic concept. Health Care Management Review, Germantown, v. 11, n. 4, p. 7-19, 1986.

BYRNE, M. M.; ASHTON, C. M. Incentives for vertical integration in healthcare: the effect of reimbursement systems. Journal of Healthcare Management, Chicago, v. 44, n. 1, p. 34-46, 1999. CONTANDRIOPOULOS, A. et al. The integration of health care: dimensions and implementation. Montreal: Université de Montreal, 2003.

DEVERS, K. J. et al. Implementing organized delivery systems: an integration scorecard. Health Care Management Review, Germantown, v. 19, n. 3, p. 7-20, 1994.

GRÖNE, O.; GARCIA-BARBERO, M. Integrated care: a position paper of the WHO European Office for Integrated Health Care Services. International Journal of Integrated Care, Utrecht, v. 1, n. 21, art. e21, 2001.

HOFMARCHER, M.; OXLEY, H.; RUSTICELLI, E. Improved health system performance through better care coordination. OECD Health Working Papers, Paris, n. 30, 2007.

INE - INSTITUTO NACIONAL DE ESTATÍSTICA. Estatísticas da população residente. Lisboa, 2018. Disponível em: <https://bit.ly/3ppseou>. Acesso em: 15 maio 2018.
LEICHSENRING, K. Developing integrated health and social care services for older persons in Europe. International Journal of Integrated Care, Berkeley, v. 4, n. 3, art. e10, 2004.

NUNES, A. Reformas na gestão hospitalar: análise dos efeitos da empresarialização. 2016. Tese (Doutorado em Administração da Saúde) Instituto Superior de Ciências Sociais e Políticas, Universidade de Lisboa, Lisboa, 2016.

NUNES, A. Tecnologias de informação e comunicação no registro de óbitos em Portugal. Journal of Health Informatics, São Paulo, v. 9, n. 4, p. 137-141, 2017.

OECD - ORGANIZAÇÃO PARA A COOPERAÇÃO E DESENVOLVIMENTO ECONÔMICO. State of health in the EU Portugal: perfil de saúde do país 2017. Bruxelas: European Observatory on Health Systems and Policies, 2018.

OMS - ORGANIZAÇÃO MUNDIAL DA SAÚDE . Integrated health services: what and why? Geneva, 2008. (Technical brief, n. 1). Disponível em: <https://bit.ly/3qX6SPD>. Acesso em: 12 maio 2018. OPAS - ORGANIZAÇÃO PAN-AMERICANA DA SAÚDE. Integrated health service delivery networks: concepts, policy options and a road map for implementation in the Americas. Washington, DC, 2011. Disponível em: <https://bit.ly/2YhzJlt>. Acesso em: 24 maio 2018.

PORTUGAL. Ministério da Saúde. Decreto-Lei $\mathrm{n}^{0}$ 207, de 9 de junho de 1999. Cria a Unidade Local de Saúde de Matosinhos. Diário da República, Lisboa, n. 133/1999, 9 jun. 1999. Série 1-A. Disponível em: <https://bit.ly/3opWYEi>. Acesso em: 20 maio 2018.

PORTUGAL. Ministério da Saúde. Portaria nº 95, de 4 de março de 2013. Aprova o regulamento do Sistema Integrado de Referenciação e de Gestão do Acesso à primeira consulta de especialidade hospitalar. Diário da República, Lisboa, n. 44/2013, 4 mar. 2013. Série 1. Disponível em: <https://bit.ly/3ctxıBN>. Acesso em: 20 maio 2018.

PORTUGAL. Ministério da Saúde. Portaria $\mathrm{n}^{0}$ 147, de 27 de abril de 2017. Regula o Sistema Integrado de Gestão do Acesso dos utentes ao 
Serviço Nacional de Saúde (SIGA SNS). Diário da República, Lisboa, n. 82/2017, 27 abr. 2017. Série 1. Disponível em: <https://bit.ly/39jTEGB>. Acesso em: 20 maio 2018.

SANTANA, R.; COSTA, C. A integração vertical de cuidados de saúde: aspectos conceptuais e organizacionais. Revista Portuguesa de Saúde Pública, Lisboa, v. 7, n. 1, p. 29-56, 2008.

SIMÕES, J. Retrato político da saúde: dependência do percurso e inovação em saúde: da ideologia ao desempenho. Coimbra: Almedina, 2004.

SIMÕES, J.; AUGUSTO, G. F.; FRONTEIRA, I. Introduction of freedom of choice for hospital outpatient care in Portugal: implications and results of the 2016 reform. Health Policy, Amsterdam, v. 121, n. 12, p. 1203-1207, 2017.

SOBCZAK, A. Opportunities for and constraints to integration of health services in Poland. International Journal of Integrated Care, Utrecht, v. 2, n. 23, 2002.
STARFIELD, B.; SHI, L.; MACINKO, J. Contribution of primary care to health systems and health. The Milbank Quaterly, New York, v. 83, n. 3, p. 457-502, 2005.

TANG, W. et al. How to build and evaluate an integrated health care system for chronic patients: study design of a clustered randomized controlled trial in rural China. International Journal of Integrated Care, Utrecht, v. 15, n. 7, 2015.

TSUTSUI, T. Implementation process and challenges for the community-based integrated care system in Japan. International Journal of Integrated Care, Utrecht, v. 14, n. 2, 2014.

ULS MATOSINHOS - UNIDADE LOCAL DE SAÚDE DE MATOSINHOS. Plano de atividades para o ano 2001. Matosinhos, 2000.

VIEIRA, E. W.; GAZZINELLI, A. Grau de integração da atenção primária à saúde de município de pequeno porte na Rede de Atenção à Saúde. Saúde e Sociedade, São Paulo, v. 26, n. 2, p. 448-461, 2017. 